\title{
How light the lepton flavor changing gauge bosons can be
}

\author{
Benedetta Belfatto $^{1,2, a}$, Zurab Berezhiani ${ }^{2,3, b}$ \\ ${ }^{1}$ Gran Sasso Science Institute, Viale Francesco Crispi 7, 67100 L'Aquila, Italy \\ 2 INFN, Laboratori Nazionali del Gran Sasso, 67010 Assergi, L'Aquila, Italy \\ ${ }^{3}$ Dipartimento di Fisica e Chimica, Università di L'Aquila, 67100 Coppito, L'Aquila, Italy
}

Received: 15 December 2018 / Accepted: 24 February 2019 / Published online: 7 March 2019

(C) The Author(s) 2019

\begin{abstract}
Spontaneous breaking of inter-family (horizontal) gauge symmetries can be at the origin of the mass hierarchy between the fermion families. The corresponding gauge bosons have flavor-nondiagonal couplings which generically induce the flavor changing phenomena, and this puts strong lower limits on the flavor symmetry breaking scales. However, for special choices of chiral horizontal symmetries the flavor changing effects can be naturally suppressed. For the sake of demonstration, we consider the case of leptonic gauge symmetry $S U(3)_{e}$ acting between the right-handed leptons and show that the respective gauge bosons can have mass in the $\mathrm{TeV}$ range, without contradicting the existing experimental limits.
\end{abstract}

1. The replication of fermion families is one of the main puzzles of particle physics. Three fermion families are in identical representations of the Standard Model (SM) gauge symmetry $S U(3) \times S U(2) \times U(1)$. Its electroweak $(\mathrm{EW})$ part $S U(2) \times U(1)$ is chiral with respect to fermion multiplets: the left-handed $(\mathrm{LH})$ leptons and quarks, $\ell_{L i}=\left(v_{i}, e_{i}\right)_{L}$ and $Q_{L i}=\left(u_{i}, d_{i}\right)_{L}$, transform as weak isodoublets while the right-handed (RH) ones $e_{R i}, u_{R i}, d_{R i}$ as isosinglets, $i=$ $1,2,3$ being the family index. The chiral fermion content of $\mathrm{SM}$ has a remarkable feature that the fermion masses emerge only after spontaneous breaking of $S U(2) \times U(1)$ by the vacuum expectation value (VEV) $\left\langle\phi^{0}\right\rangle=v_{\mathrm{w}}=174 \mathrm{GeV}$ of the Higgs doublet $\phi$, via the Yukawa couplings

$Y_{e}^{i j} \phi \overline{\ell_{L i}} e_{R j}+Y_{d}^{i j} \phi \overline{Q_{L i}} d_{R j}+Y_{u}^{i j} \tilde{\phi} \overline{Q_{L i}} u_{R j}+$ h.c.

where $Y_{e, u, d}$ are the Yukawa constant matrices, and $\tilde{\phi}=$ $i \tau_{2} \phi^{*}$. The fermion mass matrices $M_{f}=Y_{f} v_{\mathrm{w}}, f=$ $e, u, d$, can be diagonalized via bi-unitary transformations $V_{L f}^{\dagger} M_{f} V_{R f}=M_{f}^{\text {diag }}$. The masses of leptons $m_{e}, m_{\mu}, m_{\tau}$ and quarks $m_{u}, m_{d}, \ldots$ are the eigenvalues of these mass

\footnotetext{
a e-mail: benedetta.belfatto@gssi.it

b e-mail: zurab.berezhiani@lngs.infn.it
}

matrices. The "right" matrices $V_{R f}$ have no physical meaning in the SM context while the "left" ones $V_{L f}$ determine the mixing matrices in charged currents coupled to weak $W^{ \pm}$ bosons, namely $V_{\mathrm{CKM}}=V_{L u}^{\dagger} V_{L d}$ for quarks. However, no flavor mixing emerges in neutral currents coupled to $Z$ boson and Higgs boson. In this way, the SM exhibits a remarkable feature of natural suppression of flavor-changing neutral currents (FCNC) [1,2]: all FCNC phenomena are suppressed at tree level and emerge exclusively from radiative corrections. At present, the majority of experimental data on flavor changing and CP violating processes are in good agreement with the SM predictions. There are few anomalies, not definitely confirmed yet, which could point towards new physics beyond the Standard Model (BSM).

In a sense, the SM is technically natural since it can tolerate any Yukawa matrices $Y_{f}^{i j}$, but it tells nothing about their structures which remain arbitrary. So the origin of the fermion mass hierarchy and their weak mixing pattern remains a mystery.

2. The key for understanding the fermion mass and mixing pattern may lie in symmetry principles. E.g. one can assign to fermion species different charges of abelian flavor symmetry $U(1)$ [3]. Alternatively, one can introduce non-abelian horizontal gauge symmetries $G_{H}$ as e.g. $S U(3)_{H}$ [4-14] with the flavor gauge fields dynamically marking the family indices. Such a gauge theory of flavor can be considered as quantum flavordynamics, provided that it is built in a consistent way and sheds some more light on the origin of the fermion mass hierarchy.

Namely, one can envisage that the form of the Yukawa matrices in (1) is related to the VEV structures of horizontal scalar fields (known also as flavons) which spontaneously break $G_{H}$, and the fermion mass hierarchy emerges from the hierarchy between the scales of this breaking. In Refs. $[8,9]$ this conjecture was coined as hypothesis of horizontal hierarchies $(\mathrm{HHH})$. It implies that the fermion masses cannot be induced without breaking $G_{H}$ so that it cannot be 
a vector-like symmetry, but it should have a chiral character transforming the LH and RH particle species in different representations. In such a picture the fermion Yukawa couplings should emerge from the higher order "projective" operators containing flavon scalars which transfer the VEV pattern of flavons to the structure of the Yukawa matrices $Y_{f}$. In the UV-complete pictures such operators can be induced via renormalizable interactions after integrating out some extra heavy fields, scalars [4-7] or verctor-like fermions [8-14]. In the context of supersymmetry, such horizontal symmetries can lead to interesting relations between the mass spectra of fermions and their superpartners and naturally realize the minimal flavor violation scenarios [15-19].

Discovery of the flavor gauge bosons and/or related FCNC effects would point towards new BSM physics of flavor. However, a direct discovery at future accelerators can be realistic only if the scale of $G_{H}$ symmetry breaking is rather low, in the range of few $\mathrm{TeV}$. Therefore, the following questions arise: (i) for which choice of symmetry group $G_{H}$ one can realize the $\mathrm{HHH}$ paradigm, relating the fermion mass hierarchy to its breaking pattern, and (ii) which is the minimal scale of $G_{H}$ symmetry allowed by present experimental limits - namely, can this scale be low enough to have $G_{H}$ flavor bosons potentially within the experimental reach?

3. In the limit of vanishing Yukawa couplings, $Y_{f} \rightarrow 0$, the SM acquires a maximal global chiral symmetry

$U(3)_{\ell} \times U(3)_{e} \times U(3)_{Q} \times U(3)_{u} \times U(3)_{d}$

under which fermion species transform as triplets of independent $U$ (3) groups respectively as $\ell_{L} \sim 3_{\ell}, e_{R} \sim 3_{e}$, etc. The Yukawa couplings (1) can be induced by the VEVs of flavons in mixed representations of these symmetry groups. One can consider the higher order operators e.g. for leptons

$\frac{X_{e}}{M} \phi \overline{\ell_{L}} e_{R}+$ h.c.

where $X_{e} \sim\left(3_{\ell}, \overline{3}_{e}\right)$ is a flavon in mixed representation of $U(3)_{\ell} \times U(3)_{e}$ which can be also viewed as composite tensor product $3_{\ell} \times \overline{3}_{e}$ of scalars in fundamental representations of $U(3)_{\ell}$ and $U(3)_{e}$.

In the SM extensions the maximal flavor symmetry (2) reduces to a smaller symmetry. E.g. in the context of $S U(5)$ grand unified theory (GUT) which unifies $\ell_{L}$ and $d_{L}^{c}$ fragments of each family in $\overline{5}$-plets and $e_{L}^{c}, u_{L}^{c}$ and $Q_{L}$ fragments in 10-plets ( $\psi_{L}^{c}=C{\overline{\psi_{R}}}^{T}, C$ is a charge conjugation matrix), the maximal symmetry reduces to two factors $U(3)_{\ell} \times U(3)_{e}$ :

$\overline{5}_{L}=\left(\ell, d^{c}\right)_{L} \sim\left(3_{\ell}, 1\right), 10_{L}=\left(e^{c}, u^{c}, Q\right)_{L} \sim\left(1, \overline{3}_{e}\right)$

In the context of $S O(10)$ GUT all fermions of one family including the RH neutrino $N_{R}$ reside in the spinor multiplet $16_{L}=(\overline{5}+10+1)_{L}$. Hence, there can be only one chiral symmetry $U(3)$ between three families of 16-plets, with all
LH fermions $\ell_{L}, Q_{L}$ transforming as triplets and the $\mathrm{RH}$ ones $N_{R}, e_{R}, u_{R}, d_{R}$ as anti-triplets, in the spirit of chiral horizontal $S U(3)_{H}$ of Refs. [4-14]. For predictive models based on $S O(10) \times S U(3)_{H}$ see e.g. in Refs. [20-23].

It is tempting to consider some part of the maximal flavor symmetry (2), or its GUT-restricted versions, as a gauge symmetry $G_{H}$. Gauging of chiral $U(1)$ factors is difficult since they are anomalous with respect to the SM. ${ }^{1}$ Therefore, we consider a situation in which only some of non-abelian $S U$ (3) parts in (2) are gauged. In particular, in this paper we concentrate on the lepton sector and discuss a simple model with a gauge symmetry $G_{H}=S U(3)_{e}$ transforming the RH leptons as a triplet $e_{R \alpha}=\left(e_{1}, e_{2}, e_{3}\right)_{R}$, while the LH leptons $\ell_{L i}=\ell_{1,2,3}$ have no symmetry and $i=1,2,3$ is just a family number. ${ }^{2}$

We show that the lepton mass hierarchy $m_{\tau} \gg m_{\mu} \gg m_{e}$ can be directly related to the hierarchy of $U(3)_{e}$ symmetry breaking scales. As for the lepton flavor violating (LFV) phenomena induced by $S U(3)_{e}$ gauge bosons, we show that they are strongly suppressed since the intermediate $S U(2)_{e}$ subgroup acts as an approximate custodial symmetry. ${ }^{3}$ The respective scale is allowed to be as low as $2 \mathrm{TeV}$, without contradicting the present experimental limits on the LFV processes. $^{4}$

4. The LH and RH lepton fields of our model are in the following representations:

$\ell_{L i}=\left(\begin{array}{c}v_{i} \\ e_{i}\end{array}\right)_{L} \sim(2,-1,1), \quad e_{R \alpha} \sim\left(1,-2,3_{e}\right)$

where we explicitly indicate the multiplet content with respect to the EW $S U(2) \times U(1)$ and horizontal $S U(3)_{e}$. This set of fermions is not anomaly free. The ways of the anomaly cancellation will be discussed later in next sections.

We assume that there is only one Higgs doublet $\phi$ with the standard Higgs potential $V(\phi)=\lambda\left(|\phi|^{2}-v_{\mathrm{w}}^{2}\right)^{2}$. However, the Yukawa couplings of $\phi$ with the fermions $\ell_{L i}$ and $e_{R \alpha}$ are forbidden by $S U(3)_{e}$ symmetry. So, for generating the lepton masses this symmetry should be broken.

For breaking $S U(3)_{e}$ we introduce three flavon fields $\xi_{n}^{\alpha}, n=1,2,3$, each transforming as $S U(3)_{e}$ (anti)triplet.

\footnotetext{
1 However, there are models in which string-inspired anomalous gauge symmetry $U(1)_{A}$ is used as a flavor symmetry [24-29].

2 Alternatively, one could say that also $S U(3)_{\ell}$ is a gauge symmetry but broken at some higher scales. More complete model with $S U(3)_{\ell} \times$ $S U(3)_{e}$ symmetry will be discussed elsewhere [30].

3 Also the vector-like $S U$ (2) acting on both LH and RH fermion species has custodial properties [31]. However, it allows degenerate mass spectrum which makes problematic the naturalness of inter-family mass hierarchy.

4 For comparison, the naive lower limit on the scale of flavor changing bosons is over $100 \mathrm{TeV}$ [32]. In the models [4-14] this scale was assumed to be close to the GUT scale, and in any case larger than a PeV, for avoiding the excessive FCNC. For an exception, see Ref. [33].
} 

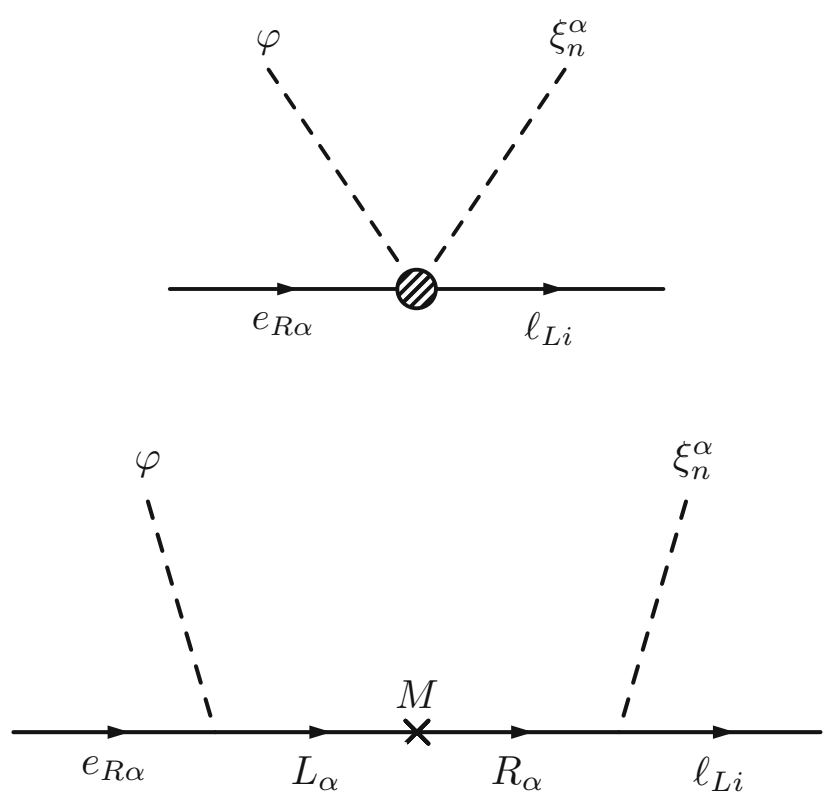

Fig. 1 Upper diagram represents operator (6) and lower diagram shows how it can be obtained via seesaw exchange of heavy vector-like fermions (8)

The charged lepton masses then can emerge from the gauge invariant dimension 5 operators

$\sum_{n} \frac{g_{i n} \xi_{n}^{\alpha}}{M} \phi \overline{\ell_{L i}} e_{R \alpha}+$ h.c.

where $g_{\text {in }}$ are order one constants (see upper diagram of Fig. 1). For having an UV-complete theory, one can consider these operators as induced from the renormalizable terms via seesaw-like mechanism [8,9]. E.g. one can integrate out from the following Yukawa Lagrangian

$h \phi \overline{L_{\alpha}} e_{R \alpha}+M \overline{R_{\alpha}} L_{\alpha}+\sum_{n} g_{i n} \xi_{n}^{\alpha} \overline{\ell_{L i}} R_{\alpha}+$ h.c.,

the extra heavy vector-like lepton doublets

$L_{\alpha}, R_{\alpha}=\left(\begin{array}{c}N_{\alpha} \\ E_{\alpha}\end{array}\right)_{L, R} \sim\left(2,-1,3_{e}\right)$

with a large Dirac mass $M$ (see Fig. 1, lower diagram).

Operator (6) has a global symmetry $U(3)_{e}=S U(3)_{e} \times$ $U(1)_{e}$, where the abelian part $U(1)_{e}$ is related to the phase change of fermions $e_{R \alpha}$ and flavons $\xi_{n}^{\alpha}$. In order to generate non-zero masses of all three leptons $e, \mu, \tau$, this global symmetry must be fully broken. This means that all three flavons $\xi_{n}$ should have the non-zero VEVs with disoriented directions. In other words, the VEVs $\left\langle\xi_{n}^{\alpha}\right\rangle$ should form a rank3 matrix, which is generically non-diagonal. Then, without loss of generality, one can choose superpositions of these fields $\xi_{n} \rightarrow U_{n m} \xi_{m}$ so that their VEVs are orthogonal and hence the matrix $\left\langle\xi_{n}^{\alpha}\right\rangle$ becomes diagonal, $\left\langle\xi_{n}^{\alpha}\right\rangle=v_{n} \delta_{n}^{\alpha}$, or in explicit form $\left\langle\xi_{1}\right\rangle=\left(\begin{array}{c}v_{1} \\ 0 \\ 0\end{array}\right), \quad\left\langle\xi_{2}\right\rangle=\left(\begin{array}{c}0 \\ v_{2} \\ 0\end{array}\right), \quad\left\langle\xi_{3}\right\rangle=\left(\begin{array}{c}0 \\ 0 \\ v_{3}\end{array}\right)$,

ordered as $v_{3}>v_{2}>v_{1}$ reflecting the steps of the global symmetry breaking $U(3)_{e} \rightarrow U(2)_{e} \rightarrow U(1)_{e} \rightarrow$ nothing. By substituting these VEVs in operator (6), it reduces to the SM Yukawa couplings

$Y_{e}^{i \alpha} \phi \overline{\ell_{L i}} e_{R \alpha}+$ h.c., $\quad Y_{e}^{i \alpha}=\sum_{n} \frac{g_{i n}\left\langle\xi_{n}^{\alpha}\right\rangle}{M}=g_{i \alpha} \frac{v_{\alpha}}{M}$.

Without loss of generality, $\ell_{L i}$ states can be turned to the basis in which matrix $g_{\text {in }}$ has a triangular form and the diagonal elements $g_{33}, g_{22}, g_{11}$ are real. Then

$$
\begin{aligned}
Y_{e} & =\frac{1}{M}\left(\begin{array}{lll}
g_{11} v_{1} & 0 & 0 \\
g_{21} v_{1} & g_{22} v_{2} & 0 \\
g_{31} v_{1} & g_{32} v_{2} & g_{33} v_{3}
\end{array}\right) \\
& =\frac{v_{3}}{M}\left(\begin{array}{lll}
g_{11} \tilde{\varepsilon} \varepsilon & 0 & 0 \\
g_{21} \tilde{\varepsilon} \varepsilon & g_{22} \varepsilon & 0 \\
g_{31} \tilde{\varepsilon} \varepsilon & g_{32} \varepsilon & g_{33}
\end{array}\right)
\end{aligned}
$$

where we denote $v_{2} / v_{3}=\varepsilon$ and $v_{1} / v_{2}=\tilde{\varepsilon}$. The Yukawa matrix $Y_{e}$ (and the mass matrix $M_{e}=Y_{e} v_{\mathrm{w}}$ ) can be diagonalized via bi-unitary transformation

$Y_{e} \rightarrow V_{L}^{\dagger} Y_{e} V_{R}=\operatorname{diag}\left(y_{e}, y_{\mu}, y_{\tau}\right)$.

Hence, modulo $\sim 1$ ratios of the Yukawa constants, the mass hierarchy $m_{\tau}: m_{\mu}: m_{e}$ corresponds to the hierarchy between the scales $v_{3}: v_{2}: v_{1}$. Namely, neglecting the small $\sim \varepsilon^{2}$ corrections, we have

$m_{\tau}=\frac{g_{33} v_{\mathrm{w}}}{M} v_{3}, \quad m_{\mu}=\frac{g_{22} v_{\mathrm{w}}}{M} v_{2}, \quad m_{e}=\frac{g_{11} v_{\mathrm{w}}}{M} v_{1}$.

Let us discuss whether such a hierarchy of the VEVs can be natural. Since three flavons have identical quantum numbers, their scalar potential has a generic form

$$
\begin{aligned}
\mathcal{V}(\xi)= & \lambda_{n}\left(\left|\xi_{n}\right|^{2}-\frac{\mu_{n}^{2}}{2 \lambda_{n}}\right)^{2}+\lambda_{k l n m} \xi_{k}^{\dagger} \xi_{l} \xi_{n}^{\dagger} \xi_{m} \\
& +\left(\mu \xi_{1} \xi_{2} \xi_{3}+\text { h.c. }\right)
\end{aligned}
$$

We assume that all constants $\lambda$ are order 1 , fluctuating say in the range $\lambda \sim 0.1 \div 1$, and the mass terms $\mu_{n}^{2}$, positive or negative, are of the same order, fluctuating say in the range of several TeV. The last (trilinear) coupling $\mu \epsilon_{\alpha \beta \gamma} \xi_{1}^{\alpha} \xi_{2}^{\beta} \xi_{3}^{\gamma}$ has a dimensional constant $\mu$ which is however allowed (by 't Hooft's naturalness principle) to be arbitrarily small since in the limit $\mu \rightarrow 0$ the Lagrangian acquires global $U(1)_{e}$ symmetry respected also by the Yukawa terms (6). In fact, this latter coupling softly breaks $U(1)_{e}$ and thus reduces the global symmetry $U(3)_{e}$ to $S U(3)_{e}$.

For full breaking of gauge $S U(3)_{e}$ symmetry, just two flavons with non-aligned VEVs are sufficient. An order of magnitude hierarchy between the scales $v_{2}$ and $v_{3}, v_{2} / v_{3} \sim$ 
$m_{\mu} / m_{\tau}$, can emerge due to some moderate conspiracy of parameters admitting a natural "spread", say within an order of magnitude, between the mass terms and coupling constants of $\xi_{2}$ and $\xi_{3}$ in (14). But large hierarchy $v_{1} / v_{3} \sim m_{e} / m_{\tau}$ at first sight requires a strong fine tuning. However, small $v_{1}$ can be obtained naturally considering the case in which the VEV matrix $\left\langle\xi_{n}^{\alpha}\right\rangle$ has rank 2 in the limit $\mu \rightarrow 0$. This means that only two flavons, $\xi_{2}$ and $\xi_{3}$, get the VEVs oriented as in (9), because of their negative mass ${ }^{2}$ terms in (14), while the third flavon $\xi_{1}$ has a positive mass ${ }^{2}$ term, i.e. $\mu_{1}^{2}<0$, and in the limit $\mu=0$ it remains VEVless. The ratio of VEVs $v_{3} / v_{2}=\left(\mu_{3} / \mu_{2}\right) \sqrt{\lambda_{2} / \lambda_{3}}$ can be of one order of magnitude due to natural fluctuation of the involved parameters. Then for $\mu \neq 0$ the last term in (14) explicitly breaks global $U(1)_{e}$ symmetry and induces non-zero VEV $\left\langle\xi_{1}\right\rangle, v_{1}=\mu v_{2} v_{3} / \mu_{1}^{2}$. Thus, taking $\mu$ small enough, say $\mu<v_{2}$, one can naturally get $v_{1} \ll v_{2}$.

The unitary matrix $V_{R}$ in (12) connecting the initial flavor basis of the RH leptons to the mass basis,

$$
\left(\begin{array}{l}
e_{1} \\
e_{2} \\
e_{3}
\end{array}\right)_{R}=V_{R}\left(\begin{array}{l}
e \\
\mu \\
\tau
\end{array}\right)_{R}=\left(\begin{array}{lll}
V_{1 e} & V_{1 \mu} & V_{1 \tau} \\
V_{2 e} & V_{2 \mu} & V_{2 \tau} \\
V_{3 e} & V_{3 \mu} & V_{3 \tau}
\end{array}\right)\left(\begin{array}{c}
e \\
\mu \\
\tau
\end{array}\right)_{R}
$$

has no physical meaning for the EW interactions, but it is meaningful for the LFV interactions mediated by the gauge bosons of $S U(3)_{e}$. Since the mixing angles in $V_{R}$ are small, we have (modulo $\varepsilon^{2}$ corrections) $V_{1 e}, V_{2 \mu}, V_{3 \tau}=1$ while for non-diagonal elements we get

$V_{3 \mu}=-\frac{g_{32}}{g_{33}} \varepsilon, \quad V_{2 e}=-\frac{g_{21}}{g_{22}} \tilde{\varepsilon}, \quad V_{3 e}=-\frac{g_{31}}{g_{33}} \tilde{\varepsilon} \varepsilon$.

The other elements in matrix $V_{R}$ can be obtained from its unitarity.

As for the mixing matrix $V_{L}$ of the LH charged leptons in (12), it is very close to unit matrix, with non-diagonal elements $\sim \varepsilon^{2}$. Therefore, the neutrino mixing angles are determined by the form of the neutrino mass matrix. The neutrino masses are induced by higher order operator [34]

$\frac{Y_{v}^{i j}}{\mathcal{M}} \phi \phi \ell_{L i}^{T} C \ell_{L j}+$ h.c..

where $\mathcal{M}$ is a new scale which in the context of seesaw mechanism can be related to the Majorana masses of RH neutrinos. In our scenario the states $\ell_{L i}$ are not distinguished by any symmetry and the matrix $Y_{v}^{i j}$ is a generic nondiagonal matrix, supposedly with all elements of the same order. Thus, the unitary matrix $V_{v}$ which diagonalizes it, $V_{\nu}^{T} Y_{\nu} V_{\nu}=Y_{v}^{\text {diag }}$, contains large rotations and the neutrino mixing angles are expected to be large.

5. Gauge bosons $\mathcal{F}_{a}^{\mu}$ of $S U(3)_{e}$, associated to the GellMann matrices $\lambda_{a}, a=1,2, \ldots 8$, interact as $g \mathcal{F}_{a}^{\mu} J_{a \mu}$ with the respective currents $J_{a \mu}=\frac{1}{2} \overline{\mathbf{e}_{R}} \lambda_{a} \gamma_{\mu} \mathbf{e}_{R}$, where $g$ is the
$S U(3)_{e}$ gauge coupling and $\mathbf{e}_{R}=\left(e_{1}, e_{2}, e_{3}\right)_{R}^{T}$ denotes the triplet of the RH leptons. Clearly, these currents, in particular those related to non-diagonal generators $\lambda_{1,2}$ etc., are generically FCNC. Nevertheless, as we shall see below, the processes mediated by flavor bosons exhibit no LFV in the initial eigenstates basis $e_{R 1}, e_{R 2}, e_{R 3}$ of the flavor diagonal generators $\lambda_{3}$ and $\lambda_{8}$.

At low energies the flavor bosons induce four-fermion (current $\times$ current) interactions:

$\mathcal{L}_{\text {eff }}=-g^{2} J_{a}^{\mu}\left(2 M^{2}\right)_{a b}^{-1} J_{b \mu}$

where $M_{a b}^{2}$ is the (symmetric) mass matrix of gauge bosons $\mathcal{F}_{a}^{\mu}$. In the flavon VEV basis (9) this matrix is essentially diagonal apart of a non-diagonal $2 \times 2$ block related to $\mathcal{F}_{3}-$ $\mathcal{F}_{8}$ mixing. Namely, for the masses of gauge bosons $\mathcal{F}_{4,5}^{\mu}$, $\mathcal{F}_{6,7}^{\mu}$ and $\mathcal{F}_{1,2}^{\mu}$ we have respectively

$M_{4,5}^{2}=\frac{g^{2}}{2}\left(v_{3}^{2}+v_{1}^{2}\right), \quad M_{6,7}^{2}=\frac{g^{2}}{2}\left(v_{3}^{2}+v_{2}^{2}\right)$,

$M_{1,2}^{2}=\frac{g^{2}}{2}\left(v_{2}^{2}+v_{1}^{2}\right)$,

while for the mass matrix of $\mathcal{F}_{3}^{\mu}-\mathcal{F}_{8}^{\mu}$ system we get

$M_{38}^{2}=\frac{g^{2}}{2}\left(\begin{array}{cc}v_{2}^{2}+v_{1}^{2} & \frac{1}{\sqrt{3}}\left(v_{1}^{2}-v_{2}^{2}\right) \\ \frac{1}{\sqrt{3}}\left(v_{1}^{2}-v_{2}^{2}\right) & \frac{1}{3}\left(4 v_{3}^{2}+v_{1}^{2}+v_{2}^{2}\right)\end{array}\right)$.

Obviously, the factor $g^{2}$ in operators (18) cancels and their strength is determined solely by $S U(3)_{e}$ symmetry breaking scales $v_{2}$ and $v_{3}$. In the following we neglect a small contribution $v_{1}^{2} / v_{2}^{2}=\tilde{\varepsilon}^{2} \ll 1$ in the gauge boson mass terms and in respective effective operators. Then

$g^{2}\left(2 M_{38}^{2}\right)^{-1}=\frac{1}{v_{2}^{2}}\left(\begin{array}{ll}1 & 0 \\ 0 & 0\end{array}\right)+\frac{1}{4 v_{3}^{2}}\left(\begin{array}{cc}1 & \sqrt{3} \\ \sqrt{3} & 3\end{array}\right)$

Hence, from all operators (18) one can single out the operator $\mathcal{L}_{2}$ which is cut off by the smaller scale $v_{2}$ :

$\mathcal{L}_{2}=-\frac{1}{v_{2}^{2}} \sum_{a=1}^{3}\left(J_{a}^{\mu}\right)^{2}=-\frac{1}{4 v_{2}^{2}} \sum_{a=1}^{3}\left(\overline{\mathbf{e}_{R}} \lambda_{a} \gamma^{\mu} \mathbf{e}_{R}\right)^{2}$

which involves only $e_{R 1}$ and $e_{R 2}$ states. Using Fierz identities for $\lambda_{1,2,3}$ which in fact are the Pauli matrices, this operator can be rewritten as

$\mathcal{L}_{2}=-\frac{1}{v_{2}^{2}}\left(J_{0}^{\mu}\right)^{2}=-\frac{1}{4 v_{2}^{2}}\left(\overline{\mathbf{e}_{R}} \lambda_{0} \gamma^{\mu} \mathbf{e}_{R}\right)^{2}$,

where we denote $\lambda_{0}=\operatorname{diag}(1,1,0)$. The remaining operators in (18) are related to the scale $v_{3}$ :

$$
\begin{aligned}
\mathcal{L}_{3}= & -\frac{1}{v_{3}^{2}}\left[\left(\tilde{J}_{3}^{\mu}\right)^{2}+\left(J_{4}^{\mu}\right)^{2}+\left(J_{5}^{\mu}\right)^{2}\right] \\
& -\frac{1}{v_{3}^{2}+v_{2}^{2}}\left[\left(J_{6}^{\mu}\right)^{2}+\left(J_{7}^{\mu}\right)^{2}\right],
\end{aligned}
$$


where the current $\tilde{J}_{3}^{\mu}=\frac{1}{2} J_{3}^{\mu}+\frac{\sqrt{3}}{2} J_{8}^{\mu}$ has a form $\frac{1}{2} \overline{\mathbf{e}}_{R} \tilde{\lambda}_{3} \gamma^{\mu} \mathbf{e}_{R}$ with $\tilde{\lambda}_{3}=\operatorname{diag}(1,0,-1)$. Hence, $\tilde{\lambda}_{3}, \lambda_{4}$ and $\lambda_{5}$ form a $S U$ (2) subalgebra of $S U(3)_{e}$, and using the Fierz identities for these matrices one can rewrite (24) as

$$
\begin{aligned}
\mathcal{L}_{3} & =-\frac{1}{v_{3}^{2}}\left[\left(\tilde{J}_{0}^{\mu}\right)^{2}+\left(J_{6}^{\mu}+i J_{7}^{\mu}\right)\left(J_{6 \mu}-i J_{7 \mu}\right)\right] \\
& =-\frac{\varepsilon^{2}}{4 v_{2}^{2}}\left[\left(\overline{\mathbf{e}_{R}} \tilde{\lambda}_{0} \gamma^{\mu} \mathbf{e}_{R}\right)^{2}+4\left(\overline{e_{R 2}} \gamma_{\mu} e_{R 2}\right)\left(\overline{e_{R 3}} \gamma^{\mu} e_{R 3}\right)\right]
\end{aligned}
$$

where $\tilde{\lambda}_{0}=\operatorname{diag}(1,0,1)$. So, operators $\mathcal{L}_{2}$ and $\mathcal{L}_{3}$ do not induce any LFV transition between $e_{R 1}, e_{R 2}, e_{R 3}$ states.

In basis of mass eigenstates $(e, \mu, \tau)$ all currents involved in operators (23) and (25), including those related to $\lambda_{0}$ and $\tilde{\lambda}_{0}$, should be rotated with the matrix $V_{R}(15)$, or in explicit form

$J_{a \mu}=(\bar{e}, \bar{\mu}, \bar{\tau})_{R} \gamma_{\mu} \frac{V_{R}^{\dagger} \lambda_{a} V_{R}}{2}\left(\begin{array}{c}e \\ \mu \\ \tau\end{array}\right)_{R}$

In particular, in $(e, \mu, \tau)$ basis $\lambda_{0}$ in operator (23) is deformed to $\lambda_{V}=V_{R}^{\dagger} \lambda_{0} V_{R}$, or explicitly

$$
\begin{aligned}
& \lambda_{V}=\left(\begin{array}{ccc}
1-\left|V_{3 e}\right|^{2} & -V_{3 e}^{*} V_{3 \mu} & -V_{3 e}^{*} V_{3 \tau} \\
-V_{3 e} V_{3 \mu}^{*} & 1-\left|V_{3 \mu}\right|^{2} & -V_{3 \mu}^{*} V_{3 \tau} \\
-V_{3 e} V_{3 \tau}^{*} & -V_{3 \mu} V_{3 \tau}^{*} & \left|V_{1 \tau}\right|^{2}+\left|V_{2 \tau}\right|^{2}
\end{array}\right) \\
& =\left(\begin{array}{ccc}
1 & \sim \tilde{\varepsilon} \varepsilon^{2} & \sim \tilde{\varepsilon} \varepsilon \\
\sim \tilde{\varepsilon} \varepsilon^{2} & 1 & \sim \varepsilon \\
\sim \tilde{\varepsilon} \varepsilon & \sim \varepsilon & \sim \varepsilon^{2}
\end{array}\right) .
\end{aligned}
$$

and $\tilde{\lambda}_{V}=V_{R}^{\dagger} \tilde{\lambda}_{0} V_{R}$ :

$$
\begin{aligned}
\tilde{\lambda}_{V} & =\left(\begin{array}{ccc}
1-\left|V_{2 e}\right|^{2} & -V_{2 e}^{*} V_{2 \mu} & -V_{2 e}^{*} V_{2 \tau} \\
-V_{2 e} V_{2 \mu}^{*} & \left|V_{1 \mu}\right|^{2}+\left|V_{3 \mu}\right|^{2} & -V_{2 \mu}^{*} V_{2 \tau} \\
-V_{2 e} V_{2 \tau}^{*} & -V_{2 \mu} V_{2 \tau}^{*} & 1-\left|V_{2 \tau}\right|^{2}
\end{array}\right) \\
& =\left(\begin{array}{ccc}
1 & \sim \tilde{\varepsilon} & \sim \tilde{\varepsilon} \varepsilon \\
\sim \tilde{\varepsilon} & \sim \varepsilon^{2} & \sim \varepsilon \\
\sim \tilde{\varepsilon} \varepsilon & \sim \varepsilon & 1
\end{array}\right) .
\end{aligned}
$$

where we indicate the order of magnitude for the LFV entries in terms of the small parameters $\varepsilon$ and $\tilde{\varepsilon}$.

6. Let us question now how small the scale $v_{2}$ can be without contradicting to existing experimental limits. The leading terms in (23) give rise to flavor conserving operators

$$
-\frac{1}{4 v_{2}^{2}}\left(\overline{e_{R}} \gamma^{v} e_{R}\right)^{2}-\frac{1}{2 v_{2}^{2}}\left(\overline{e_{R}} \gamma^{v} e_{R}\right)\left(\overline{\mu_{R}} \gamma_{v} \mu_{R}\right)
$$

constrained by the compositeness limits $\Lambda_{R R}^{-}($eeee $)>$ $10.2 \mathrm{TeV}$ and $\Lambda_{R R}^{-}(e e \mu \mu)>9.1 \mathrm{TeV}(95 \%$ C.L.) as reported respectively in Refs. [35] and [36]. Translating the formal definitions of compositeness scales to the scale $v_{2}$, we get

$$
\begin{aligned}
& v_{2}=(8 \pi)^{-1 / 2} \Lambda_{R R}^{-}(\text {eеeе })>2.0 \mathrm{TeV} \\
& v_{2}=(8 \pi)^{-1 / 2} \Lambda_{R R}^{-}(\text {eе } \mu \mu)>1.8 \mathrm{TeV}
\end{aligned}
$$

Hence, these limits allow the scale of $S U(2)_{e}$ symmetry to be as small as $v_{2}=2 \mathrm{TeV}$. Using Eq. (13), then one can evaluate also the scale of $S U(3)_{3} \rightarrow S U(2)_{e}$ breaking $v_{3}=v_{2} / \varepsilon$ and the cutoff scale $M$ as

$v_{3} \simeq\left(g_{22} / g_{33}\right) \times 34 \mathrm{TeV}, \quad M \simeq g_{22} \times 3.3 \mathrm{PeV}$.

As for the flavor-changing phenomena induced by operators (23) and (25), they are suppressed by small parameters $\varepsilon$ and $\tilde{\varepsilon}$ and agree with severe experimental limits on the LFV even for such a low scale of flavor symmetry. E.g. both $\mathcal{L}_{2}$ and $\mathcal{L}_{3}$ terms contribute to the following operator which induces the LFV decay $\mu \rightarrow e e \bar{e}$ :

$$
\begin{aligned}
& \frac{4 G_{\mu e e e}}{\sqrt{2}}\left(\overline{e_{R}} \gamma_{\nu} \mu_{R}\right)\left(\overline{e_{R}} \gamma^{v} e_{R}\right), \\
& \frac{4 G_{\mu e e e}}{\sqrt{2}}=\frac{1}{2 v_{2}^{2}} V_{3 e}^{*} V_{3 \mu}+\frac{1}{2 v_{3}^{2}} V_{2 e}^{*} V_{2 \mu} \sim \frac{\varepsilon^{2} \tilde{\varepsilon}}{2 v_{2}^{2}},
\end{aligned}
$$

Its amplitude can be normalized to the amplitude of the muon standard decay due to the weak interactions

$-\frac{4 G_{F}}{\sqrt{2}}\left(\overline{e_{L}} \gamma_{\rho} v_{e}\right)\left(\overline{v_{\mu}} \gamma^{\rho} \mu_{L}\right)$

where $4 G_{F} / \sqrt{2}=1 / v_{\mathrm{w}}^{2}, v_{\mathrm{w}}=174 \mathrm{GeV}$. Hence, we get

$$
\frac{\Gamma(\mu \rightarrow e e \bar{e})}{\Gamma\left(\mu \rightarrow e \bar{v}_{e} v_{\mu}\right)}=\frac{1}{2}\left|\frac{G_{\mu e e e}}{G_{F}}\right|^{2} \sim \frac{\tilde{\varepsilon}^{2} \varepsilon^{4}}{8}\left(\frac{v_{\mathrm{w}}}{v_{2}}\right)^{4} \text {. }
$$

Hence, for $v_{2}>2 \mathrm{TeV}$ and $\varepsilon, \tilde{\varepsilon} \leq 1 / 20$ or so, this branching ratio is compatible with the existing experimental limit $\operatorname{Br}(\mu \rightarrow 3 e)_{\exp }<10^{-12}$ [37].

For $\tau$ lepton decay modes as $\tau \rightarrow \mu e \bar{e}$ and $\tau \rightarrow 3 \mu$ leading contributions arise from operator (23). From (27) we get the relevant constants as $4 G_{\tau \mu e e} \sqrt{2}=4 G_{\tau \mu \mu \mu} / \sqrt{2}=$ $V_{3 \mu}^{*} V_{3 \tau} / 2 v_{2}^{2}$. Hence, the widths of these decays are suppressed by a factor $\sim \varepsilon^{2} / v_{2}^{4}$, and are compatible with the experimental limits [37]. The summary of predicted branching ratios of relevant LFV processes compared with experimental limits is given in Table 1 , with the parameters $\varepsilon, \tilde{\varepsilon}$ normalized to a benchmark value $1 / 20$.

Yet another LFV effect to be considered is muoniumantimuonium conversion $M(\bar{\mu} e) \rightarrow \bar{M}(\mu \bar{e})$ [38]. The relevant operator emerges from (25) and it reads

$$
-\frac{4 G_{M} \bar{M}}{\sqrt{2}}\left(\bar{\mu}_{R} \gamma^{v} e_{R}\right)^{2}, \quad G_{M \bar{M}}=\frac{\varepsilon^{2}\left(V_{2 e} V_{2 \mu}^{*}\right)^{2}}{8 \sqrt{2} v_{2}^{2}} .
$$

Thus the amplitude of $M-\bar{M}$ transition is doubly suppressed, by a factor $\sim \tilde{\varepsilon}^{2} \varepsilon^{2}<10^{-5}$ or so, and is much below the experimental limit $\left|G_{M \bar{M}} / G_{F}\right|<3 \times 10^{-3}$ [39]. 
Table 1 Experimental limits on the branching fractions for the LFV decays [37] vs. those predicted in our model. For the LFV decays of $\tau$ lepton the branching ratio $\operatorname{Br}\left(\tau \rightarrow \mu \nu_{\tau} \bar{v}_{\mu}\right)=0.174$ is taken into account. In last column we used Eq. (16) for the elements of mixing matrix $V_{R}$ and set the scale $v_{2}=2 \mathrm{TeV}$ from the compositeness limits.
Both parameters $\varepsilon$ and $\tilde{\varepsilon}$ are normalised to $1 / 20 \simeq m_{\mu} / m_{\tau} ; \varepsilon_{20}=20 \varepsilon$, $\tilde{\varepsilon}_{20}=20 \tilde{\varepsilon}$. The latter choice is a clear overestimation and it would be more consistent to take $\tilde{\varepsilon} \simeq m_{e} / m_{\mu} \simeq 1 / 200$. However, we see that all of the LFV limits are respected even with strongly overestimated $\tilde{\varepsilon}$

\begin{tabular}{llll}
\hline LFV mode & Experimental Br. & Predicted Br. & \\
\hline$\mu \rightarrow e e \bar{e}$ & $<1.0 \times 10^{-12}$ & $\frac{1}{8}\left(\frac{v_{\mathrm{w}}}{v_{2}}\right)^{4}\left|V_{3 e}^{*} V_{3 \mu}+\varepsilon^{2} V_{2 e}^{*} V_{2 \mu}\right|^{2}$ & $\leq 1.1 \times 10^{-13}\left(\left|\frac{g_{31}^{*} g_{32}}{g_{33}^{2}}\right|+\left|\frac{g_{21}^{*}}{g_{22}}\right|\right)^{2} \tilde{\varepsilon}_{20}^{2} \varepsilon_{20}^{4}\left(\frac{2 \mathrm{TeV}}{v_{2}}\right)^{4}$ \\
$\tau \rightarrow \mu e \bar{e}$ & $<1.8 \times 10^{-8}$ & $\frac{1}{4}\left(\frac{v_{\mathrm{w}}}{v_{2}}\right)^{4}\left|V_{3 \mu}^{*} V_{3 \tau}\right|^{2} \operatorname{Br}\left(\tau \rightarrow \mu v_{\tau} \bar{v}_{\mu}\right)$ & $=6.2 \times 10^{-9}\left|\frac{g_{32}}{g_{33}}\right|^{2} \varepsilon_{20}^{2}\left(\frac{2 \mathrm{TeV}}{v_{2}}\right)^{4}$ \\
$\tau \rightarrow \mu \mu \bar{\mu}$ & $<2.1 \times 10^{-8}$ & $\frac{1}{8}\left(\frac{v_{\mathrm{w}}}{v_{2}}\right)^{4}\left|V_{3 \mu}^{*} V_{3 \tau}\right|^{2} \mathrm{Br}\left(\tau \rightarrow \mu v_{\tau} \bar{v}_{\mu}\right)$ & $=3.1 \times 10^{-9}\left|\frac{g_{32}}{g_{33}}\right|^{2} \varepsilon_{20}^{2}\left(\frac{2 \mathrm{TeV}}{v_{2}}\right)^{4}$ \\
$\mu \rightarrow e \gamma$ & $<4.2 \times 10^{-13}$ & $\frac{3 \alpha}{2 \pi}\left(\frac{v_{\mathrm{w}}}{v_{2}}\right)^{4}\left|V_{3 e}^{*} V_{3 \mu}\right|^{2}$ & $=3.1 \times 10^{-15}\left|\frac{g_{31}^{*} g_{32}^{2}}{g_{33}^{2}}\right|^{2} \tilde{\varepsilon}_{20}^{2} \varepsilon_{20}^{4}\left(\frac{2 \mathrm{TeV}}{v_{2}}\right)^{4}$ \\
$\tau \rightarrow \mu \gamma$ & $<4.4 \times 10^{-8}$ & $\frac{3 \alpha}{2 \pi}\left(\frac{v_{\mathrm{w}}}{v_{2}}\right)^{4}\left|V_{3 \mu}^{*} V_{3 \tau}\right|^{2} \operatorname{Br}\left(\tau \rightarrow \mu v_{\tau} \bar{v}_{\mu}\right)$ & $=8.7 \times 10^{-11}\left|\frac{g_{32}}{g_{33}}\right|^{2} \varepsilon_{20}^{2}\left(\frac{2 \mathrm{TeV}}{v_{2}}\right)^{4}$
\end{tabular}

One loop contribution of flavor bosons to the electron magnetic moment has no suppression by mixing angles in $V_{R}$ (the electric dipole moment gets no contribution at one loop). By computing parameter $a_{e}=\frac{1}{2}\left(g_{e}-2\right)$ with formulas in Ref. [40], we get:

$a_{e}=-\frac{m_{e}^{2}}{8 \pi^{2} v_{2}^{2}}=-8.3 \times 10^{-16}\left(\frac{2 \mathrm{TeV}}{v_{2}}\right)^{2}$

which is about 3 orders of magnitude smaller than the present difference between the experimental [37] and theoretical [41] determinations of the electron anomalous magnetic moment, $a_{e}^{\text {exp }}-a_{e}^{\mathrm{SM}}=(-7.0 \pm 3.5) \times 10^{-13}$. Similarly, the contribution for muon anomalous magnetic moment obtained by substituting $m_{e} \rightarrow m_{\mu}$ in (36), $a_{\mu}=-3.5 \times 10^{-11}\left(2 \mathrm{TeV} / v_{2}\right)^{2}$, is two orders of magnitude below the existing discrepancy $a_{\mu}^{\exp }-a_{\mu}^{\mathrm{SM}}=(2.7 \pm 0.8) \times 10^{-9}$ [37]. So, these contributions are irrelevant for both electron and muon.

Let us remark that potentially also flavons can mediate the LFV processes. From the effective operators (6), after substituting the VEV $\langle\phi\rangle=v_{\mathrm{w}}$ we obtain for the lepton Yukawa couplings with the flavon fields $\xi_{n}$ :

$h_{i n} \xi_{n}^{\alpha} \overline{\ell_{L i}} e_{R \alpha}, \quad h_{i n}=\frac{g_{i n} v_{\mathrm{w}}}{M}$

which are generically flavor-changing. For example, in the basis (11) the Higgs mode of the flavon $\xi_{2}$ which is presumably the lightest, with the mass $\mu_{2} \sim v_{2}$, induces the following effective operator:

$$
-\frac{h_{32} h_{22}}{\mu_{2}^{2}}(\bar{\tau} \mu)(\bar{\mu} \mu), \quad \frac{h_{32} h_{22}}{\mu_{2}^{2}} \simeq \frac{m_{\mu}^{2}}{v_{2}^{4}}
$$

where we have taken into account the relations (13). Thus, for $v_{2}>2 \mathrm{TeV}$, the width of $\tau \rightarrow 3 \mu$ decay induced by this operator is more than 12 orders of magnitude below the experimental limit. The width of $\mu \rightarrow 3 e$ decay induced by analogous operator mediated by flavon $\xi_{1}$ is also suppressed by orders of magnitude.

7. Let us remark that for promoting the chiral non-abelian factors in (2) as $S U(3)_{e}$, etc. as gauge symmetries, one has to take care of anomaly cancellations. For more generality, let us consider

$S U(3)_{\ell} \times S U(3)_{e} \times S U(3)_{Q} \times S U(3)_{u} \times S U(3)_{d}$

as maximal gauge symmetry under which the different fermion species form the triplets of independent $S U(3)$ horizontal groups respectively as

$\ell_{L} \sim 3_{\ell}, e_{R} \sim 3_{e}, Q_{L} \sim 3_{Q}, u_{R} \sim 3_{u}, d_{R} \sim 3_{d}$.

Then each of these gauge factors would have triangle $S U(3)^{3}$ anomalies. For their cancellation, for each triplet in (40) one must additionally introduce ad hoc fermions of the opposite chiralities which are singlets of the SM and are triplets under respective horizontal symmetry.

Most interesting possibility is to introduce mirror sector [42-47] as a mirror copy of the SM gauge symmetry $S U(3) \times S U(2) \times U(1)$, so that for every ( $\mathrm{LH}$ or $\mathrm{RH})$ fermion species of the SM, there exists its mirror twin with the opposite chirality in the identical representation of the mirror SM' gauge group $S U(3)^{\prime} \times S U(2)^{\prime} \times U(1)^{\prime}$ (for a review see e.g. Refs. [48,49]), and assume that the gauge horizontal symmetries (39) are common symmetries between ordinary and mirror particles as it was suggested in Refs. [15,16]. In other words, for ordinary quark and lepton species in (40), their mirror twins ('primed' quarks and leptons) should be respectively in representations

$\ell_{R}^{\prime} \sim 3_{\ell}, e_{L}^{\prime} \sim 3_{e}, Q_{R}^{\prime} \sim 3_{Q}, u_{L}^{\prime} \sim 3_{u}, d_{L}^{\prime} \sim 3_{d}$.

In this picture, the parity can be understood as a discrete symmetry of exchange between ordinary and mirror species, $\ell_{L} \leftrightarrow \ell_{R}^{\prime}$ etc. with respective exchange of ordinary and mirror gauge bosons and Higgses $\phi$ and $\phi^{\prime}$. As for the horizontal 
gauge factors (39), they in fact all become vector-like, with their triangle anomalies reciprocally cancelled between the ordinary (40) and mirror (41) particle species of the opposite chiralities.

In particular, in our model "reduced" to leptons in which only $S U(3)_{e}$ is considered as a gauge symmetry, triangle $S U(3)_{e}^{3}$ anomaly is cancelled between the ordinary $\mathrm{RH}$ leptons $e_{R \alpha} \sim\left(1,-2,3_{e}\right)$ and their LH mirror partners $e_{L \alpha}^{\prime} \sim\left(1,-2^{\prime}, 3_{e}\right)$, where $-2^{\prime}$ denotes $U(1)^{\prime}$ hypercharge of mirror leptons.

Operators (6) must be complemented by similar couplings of flavons $\xi_{n}$ with mirror leptons. Hence, we have

$$
\sum_{n} \frac{g_{i n} \xi_{n}^{\alpha}}{M}\left(\phi \overline{\ell_{L i}} e_{R \alpha}+\phi^{\prime} \overline{\ell_{R i}^{\prime}} e_{L \alpha}^{\prime}\right)+\text { h.c. }
$$

Then, if mirror symmetry is exact, i.e. $\left\langle\phi^{\prime}\right\rangle=\langle\phi\rangle=v_{\mathrm{w}}$, the ordinary and mirror leptons should have identical mass spectra. As for neutrinos, now besides the operator (17) generating the neutrino Majorana masses, we should have its mirror copy generating Majorana masses of mirror neutrinos, and also a mixed operator between ordinary $\ell$ and mirror $\ell^{\prime}$ leptons [50-53]:

$$
\begin{gathered}
\frac{Y_{v}^{i j}}{\mathcal{M}}\left(\phi \phi \ell_{L i}^{T} C \ell_{L j}+\phi^{\prime} \phi^{\prime} \ell_{R i}^{\prime T} C \ell_{R j}^{\prime}\right) \\
+\frac{\tilde{Y}_{v}^{i j}}{\mathcal{M}} \phi \phi^{\prime} \overline{\ell_{L i}} \ell_{R j}^{\prime}+\text { h.c.. }
\end{gathered}
$$

The last operator mixes ordinary (active) and mirror (sterile) neutrinos, and also can play a key role in co-leptogenesis scenario which can generate baryon asymmetries in both ordinary and mirror sectors $[54,55]$. Interestingly, if lepton numbers (or better $\mathrm{B}-\mathrm{L}$ and $\mathrm{B}^{\prime}-\mathrm{L}^{\prime}$ ) are conserved in each sector, then these operators are forbidden and all neutrinos remain massless. However, if the combination $(B-L)+\left(B^{\prime}-L^{\prime}\right)$ is conserved, then the last operator is allowed. In this case the neutrinos will be Dirac particles having masses $\sim v_{\mathrm{w}}^{2} / \mathcal{M}$, with their LH components living in ordinary world and the RH components living in mirror world.

Introduction of the mirror fermions does not fully solves the anomaly problem: there remains a mixed triangle anomaly of hypercharge-flavor $U(1) \times S U(3)_{e}^{2}$. For its cancellation, new fermion species should be introduced in the proper representations of the $\mathrm{SM}$ and $S U(3)_{e}$. There are several ways of doing this. Let us consider one of the possibilities by introducing in our sector, in addition to the regular leptons (5), the new lepton species in representations

$\mathcal{E}_{L \alpha} \sim\left(1,-2,3_{e} ; X\right), \quad \mathcal{E}_{R i} \sim(1,-2,1 ; X)$,

and, for mirror parity, analogous species in mirror sector:

$\mathcal{E}_{R \alpha}^{\prime} \sim\left(1,-2^{\prime}, 3_{e} ; X\right), \quad \mathcal{E}_{L i}^{\prime} \sim\left(1,-2^{\prime}, 1 ; X\right)$, where $\alpha=1,2,3$ is a gauge $S U(3)_{e}$ index and $i=1,2,3$ is just for numbering three species. We assign to these fermions a new charge $X$ of additional gauge symmetry $U(1)_{X}$ while ordinary leptons have no $X$-charges. This additional charge is introduced in order to forbid the mixing of new fermions (44) with ordinary leptons (5) due to the mass term $M \overline{\mathcal{E}_{L \alpha}} e_{R \alpha}$ and the Yukawa terms $\overline{\ell_{L i}} \mathcal{E}_{R j} \phi$ which would ruin the flavor structure induced by the operator (6). It is easy to check that by introducing extra fermions (44) and (45) the mixed triangle anomalies including $U(1) \times S U(3)_{e}^{2}, U(1)_{X} \times S U(3)_{e}^{2}$, $U(1) \times U(1)_{X}^{2}$ and $U(1)_{X} \times U(1)^{2}$ are all cancelled.

The new fermions get masses from their Yukawa couplings with flavons $\xi_{n}$ :

$y_{i n} \xi_{n}^{\alpha} \overline{\mathcal{E}_{R i}} \mathcal{E}_{L \alpha}+y_{i n} \xi_{n}^{\alpha} \overline{\mathcal{E}_{L i}^{\prime}} \mathcal{E}_{R \alpha}^{\prime}+$ h.c.

where $y_{\text {in }}$ are order 1 Yukawa constants. Therefore, their mass spectrum should reflect the hierarchy $v_{3}: v_{2}: v_{1} \sim$ $1: \varepsilon: \varepsilon \tilde{\varepsilon}$. In particular, if $v_{2}$ is in the TeV range, then $v_{1}$ should be in the range of $100 \mathrm{GeV}$ and thus the lightest of new leptons will have a mass of this order. In addition, if $U(1)_{X}$ symmetry is unbroken, then the lightest of these states should be stable (the heavier ones will decay into the lighter one via $S U(3)_{e}$ flavor boson mediated operators). Interestingly, the LEP direct experimental lower limit on the mass of new charged leptons is $102.6 \mathrm{GeV}$ [56]. Such heavy leptons can be within the reach of new $e^{+} e^{-}$machines as ILC/CLIC or CEPC/FCC-ee. If $U(1)_{X}$ symmetry is spontaneously broken, then the mixing of $e, \mu, \tau$ with new leptons can be allowed and thus the latter will be rendered unstable.

Let us turn to flavor gauge bosons of $S U(3)_{e}$ which now interact with both normal leptons and mirror leptons. Now their exchange should create mixed effective operators involving both ordinary and mirror leptons. In particular, the bosons $\mathcal{F}_{1,2,3}^{\mu}$ mediate the following LFV operators involving first two families of both sectors:

$$
\frac{1}{v_{2}^{2}} \sum_{a=1}^{3} J_{a}^{\mu} J_{a \mu}^{\prime}=\frac{1}{4 v_{2}^{2}} \sum_{a=1}^{3}\left(\overline{\mathbf{e}_{R}} \lambda_{a} \gamma^{\mu} \mathbf{e}_{R}\right)\left(\overline{\mathbf{e}_{L}^{\prime}} \lambda_{a} \gamma_{\mu} \mathbf{e}_{L}^{\prime}\right)
$$

Thus, this operator induces muonium - mirror muonium conversion $M(\bar{\mu} e) \rightarrow M^{\prime}\left(\mu^{\prime} \bar{e}^{\prime}\right)$ with $G_{M M^{\prime}} / G_{F}=\left(v_{\mathrm{w}} / v_{2}\right)^{2}=$ $7.6 \times 10^{-3}\left(2 \mathrm{TeV} / v_{2}\right)^{2}$. In difference from the muoniumantimuonium conversion (35), here is no suppression by small mixing angles. The present limit on the muonium disappearance reads $\operatorname{Br}(M \rightarrow$ invisible $)<5.7 \times 10^{-6}$ [57] which is clearly respected for $v_{2}=2 \mathrm{TeV}$. However, this limit can be improved by several orders of magnitude as discussed in Ref. [57]. Analogously, this operator should induce positronium conversion into mirror positronium [58,59], but for $v_{2}=2 \mathrm{TeV}$ the positronium disappearance rate is much below the present experimental limit $\mathrm{Br}(\mathrm{o}-\mathrm{Ps} \rightarrow$ invisible) $<6 \times 10^{-4}[60]$. 
8. In this paper we discussed phenomenological implications of horizontal gauge symmetry $S U(3)_{e}$ acting only in lepton sector, between three families of right-handed leptons. The lepton mass hierarchy $m_{\tau} \gg m_{\mu} \gg m_{e}$ can be related to the hierarchy of the symmetry breaking scales $v_{3} \gg v_{2} \gg v_{1}$. We have shown that the LFV effects induced by flavor changing gauge bosons are strongly suppressed due to custodial properties of $S U(2)_{e} \subset S U(3)_{e}$ symmetry and respective scale can be as small as $v_{2}=2 \mathrm{TeV}$. This limit is in fact set from the compositeness limits on the flavor-conserving operators while the limits obtained from the LFV processes itself are weaker. Taken into account that the gauge coupling constant $g$ of horizontal $S U(3)_{e}$ can be less than 1 , then masses of the $S U(2)_{e}$ gauge bosons $M_{1,2,3} \simeq(g / \sqrt{2}) v_{2}$ can be as small as $1 \mathrm{TeV}$ or even smaller, and thus can be accessible at new electron-positron machines.

Analogously to $S U(3)_{e}$, all $S U$ (3) factors in (39) can be rendered anomaly free along the lines discussed in previous section, and thus they also can be gauge symmetries. ${ }^{5}$ The quark mass hierarchy can be related to hierarchies in breaking of $S U(3)_{Q} \times S U(3)_{d} \times S U(3)_{u}$ gauge factors, i.e. with the ratios $\varepsilon_{d}=v_{2}^{d} / v_{3}^{d}$ and $\tilde{\varepsilon}_{d}=v_{1}^{d} / v_{2}^{d}$ between the VEVs of $S U(3)_{d}$ triplet flavons, and the same for $S U(3)_{u}$ and $S U(3)_{Q}$. In this way, the hierarchy of down quark masses will go parametrically as $1: \varepsilon_{d} \varepsilon_{Q}: \varepsilon_{d} \tilde{\varepsilon}_{d} \varepsilon_{Q} \tilde{\varepsilon}_{Q}$ and of the up quarks as $1: \varepsilon_{u} \varepsilon_{Q}: \varepsilon_{u} \tilde{\varepsilon}_{u} \varepsilon_{Q} \tilde{\varepsilon}_{Q}$. The quark flavor violating processes mediated by gauge bosons of $S U(3)_{d}$ and $S U(3)_{u}$ will be suppressed due to custodial symmetry in the same way as the LFV processes mediated by $S U(3)_{e}$ bosons. In particular, the operator $\left(\overline{s_{R}} \gamma^{\mu} d_{R}\right)^{2}$ which induces $K^{0}-\bar{K}^{0}$ oscillation (analogously as leptonic operator (35) induces $M-\bar{M}$ conversion) will be suppressed by a factor $\sim \varepsilon_{d}^{2} \tilde{\varepsilon}_{d}^{2} \ll 1$. This can allow quark flavor changing gauge bosons to have masses in the range of few $\mathrm{TeV}$, in fact limited only by the quark compositeness bounds. Interestingly, the flavor bosons of $S U(3)_{\ell}$ and $S U(3)_{Q}$ can give also anomalous contributions imitating the charged current $\times$ current operators of the SM, and so they will have interference with the latter. E.g. $S U(3)_{\ell}$ bosons induce operator $\left(\overline{e_{L}} \gamma_{\rho} \mu_{L}\right)\left(\overline{v_{\mu}} \gamma^{\rho} \nu_{e}\right)$ which is nothing but the Fierz-transformed SM operator (33) responsible for the muon decay. Analogously, $S U(3)_{Q}$ bosons should induce e.g. operator $\left(\overline{u_{L}} \gamma_{\rho} c_{L}\right)\left(\overline{s_{L}} \gamma^{\rho} d_{L}\right)$ which also interferes with the charged current operators in the SM. Presence of such operators can affect the unitarity tests of the CKM mixing of quarks. Detailed analysis of these issues will be given elsewhere [30].

As far as the presence of mirror sector is concerned, mirror matters is a viable candidate for light dark matter dominantly

\footnotetext{
5 Moreover, also some anomaly free combinations of $U(1)$ factors in (2) can be promoted as gauge symmetries, as e.g. common $U(1)_{\mathrm{B}-\mathrm{L}}$ acting between ordinary and mirror sectors $[61,62]$.
}

consisting of mirror helium and hydrogen atoms [63-66]. The flavor gauge bosons interacting with both ordinary and mirror fermions appear as messengers between two sectors and can give an interesting portal for mirror matter direct detection, complementary to the dark photon portal related to the photon-mirror photon kinetic mixing $[67,68]$. However, they will also give rise to the mixing between the neutral ordinary and mirror mesons. Namely, the lighter flavor bosons induce mixings as $\pi^{0}-\pi^{0 \prime}, K^{0}-K^{0 \prime}$, etc. [48,49], with implications for the invisible decay channels of neutral mesons (for a recent discussion, see also Ref. [69]). In the supersymmetric version the respective flavor gauginos, complemented by R-parity breaking, can induce the mixing between the ordinary and mirror neutral baryons. Interestingly, neutron-mirror neutron oscillation $n-n^{\prime}$ related to physics at the scale of few $\mathrm{TeV}$ [70-72] can be rather fast, in fact much faster than the neutron decay itself. Recent summary of experimental bounds on the $n-n^{\prime}$ oscillation time can be found in Ref. [73]). There are some anomalies in existing experiments on $n-n^{\prime}$ oscillation search [74] which can be tested in planned experiments on the neutron disappearance and regeneration $[75,76]$.

Oscillation phenomena between ordinary and mirror neutral particles are effective if they are degenerate in mass, i.e. mirror parity is unbroken and the weak scales $\langle\phi\rangle=v_{\mathrm{w}}$ and $\left\langle\phi^{\prime}\right\rangle=v_{\mathrm{w}}^{\prime}$ are exactly equal in two sectors, $v_{\mathrm{w}}^{\prime}=v_{\mathrm{w}}$. However, the cancellation of horizontal anomalies between two sectors does not require that mirror parity is unbroken, and in fact one can consider models where it is spontaneously broken, e.g. $v_{\mathrm{w}}^{\prime}>v_{\mathrm{w}}$, with interesting implications for mirror dark matter properties and sterile mirror neutrinos [77] and axion physics [78]. In particular, in the context of the mirror twin Higgs mechanism for solving the little hierarchy problem, in supersymmetric $[79,80]$ or non-supersymmetric [81] versions, one expects $v_{\mathrm{w}}^{\prime}$ in the TeV range. Summarizing all, $\mathrm{TeV}$ scale still remains as a realistic scale for consistent supersymmetric models [82]. Supersymmetric extension of our model with $\mathrm{TeV}$ scale horizontal gauge symmetry can be a part of this picture as far as it is not problematic with respect to dangerous flavor-changing phenomena. It can be built in straightforward way, just by promoting all involved fields as superfields of corresponding chiralities and associating respectively the Yukawa terms (7) etc. with the superpotential terms. Moreover, this framework allows to realize MFV paradigm providing a theoretical motivation for the alignment of the fermion and sfermion masses along the lines discussed in Refs. [15-19]. In particular, in supersymmetrized version of our model the soft LFV breaking terms $B_{e}^{i \alpha} \phi \tilde{\ell}_{L i}^{\dagger} \tilde{e}_{R \alpha}$, where tildes mark the slepton fields, in the leading approximation will be aligned to the Yukawa terms (10), $B_{e}^{i \alpha} \propto Y_{e}^{i \alpha}$. Thus, for the slepton masses in the TeV range, the contributions of these soft trilinear terms to the LFV 
phenomena as $\mu \rightarrow e \gamma, \tau \rightarrow \mu \gamma$, etc. will be properly suppressed.

The following remark is in order. The viability of mirror sector is subject of strong cosmological restrictions. Namely, the Big Bang nucleosynthesis (BBN) constraints require that at the BBN epoch its temperature should be smaller than the temperature of the ordinary sector, $T^{\prime} / T<0.6$ [63]. The constraints from the $\mathrm{CMB}$ and large scale structure are at least twice stronger, $T^{\prime} / T<0.2 \div 0.3[65,66]$. On the other hand, the interactions (47) induce the process $e \bar{e} \rightarrow e^{\prime} \bar{e}^{\prime}$ which in the early universe would bring two sectors into equilibrium. The freeze-out temperature $T_{d}$ of this process can be easily estimated, just by rescaling by a factor $\left(2 v_{2} / v_{\mathrm{w}}\right)^{4 / 3}$ the neutrino decoupling temperature $T_{v} \simeq 2 \mathrm{MeV}$. Thus, for respecting the cosmological bounds, the reheating temperature of the universe should not exceed $T_{d} \simeq\left(v_{2} / 2 \mathrm{TeV}\right)^{4 / 3} \times 130 \mathrm{MeV}$. (Analogous problem of low reheating temperature is typical also for the $\mathrm{TeV}$ scale gravity models with large extra dimensions, as discussed in Ref. [83], and for twin Higgs models [79-81].) Alternatively, one has to assume a possibility of additional entropy production in ordinary sector below the temperatures $T_{d}$ which in turn implies the necessity of mirror symmetry breaking. In asymmetric mirror model, with $v_{\mathrm{w}}^{\prime} \gg v_{\mathrm{w}}, T_{d}$ becomes significantly larger. In particular, for $v_{\mathrm{w}}^{\prime}$ larger than few $\mathrm{PeV}$, as e.g. in heavy axion (axidragon) model [78], one can have $T_{d}>1 \mathrm{TeV}$ or so.

Concluding, in this paper we demonstrated that in the $\mathrm{TeV}$ range there may exist a new physics related to the fermion flavor which can be revealed in future experiments at the energy and precision frontiers. In particular, the lepton-flavor changing gauge bosons can be as light as one $\mathrm{TeV}$ or even lighter, since the LFV processes are strongly suppressed by custodial symmetry. Nevertheless, some of these LFV processes, as e.g. $\tau \rightarrow 3 \mu$, can have widths close to present experimental limits and can be within the reach of future high precision experiments.

The work of Z.B. was partially supported by Shota Rustaveli National Science Foundation of Georgia, Grant DI-18335/New Theoretical Models for Dark Matter Exploration. Preliminary version of this work was presented by B.B. at the European Physical Society Conference on High Energy Physics EPS-HEP 2017 [84].

Data Availability Statement This manuscript has no associated data or the data will not be deposited. [Authors' comment: This is a theoretical work. No experimental data were used.]

Open Access This article is distributed under the terms of the Creative Commons Attribution 4.0 International License (http://creativecomm ons.org/licenses/by/4.0/), which permits unrestricted use, distribution, and reproduction in any medium, provided you give appropriate credit to the original author(s) and the source, provide a link to the Creative Commons license, and indicate if changes were made.

Funded by SCOAP ${ }^{3}$.

\section{References}

1. S.L. Glashow, S. Weinberg, Phys. Rev. D 15, 1958 (1977)

2. E.A. Paschos, Phys. Rev. D 15, 1966 (1977)

3. C.D. Froggatt, H.B. Nielsen, Nucl. Phys. B 147, 277 (1979)

4. Z. Berezhiani, J.L. Chkareuli, Sov. J. Nucl. Phys. 37, 618 (1983)

5. Z. Berezhiani, J.L. Chkareuli, JETP Lett. 35, 612 (1982)

6. Z. Berezhiani, J.L. Chkareuli, JETP Lett. 37, 338 (1983)

7. Z. Berezhiani, J.L. Chkareuli, Sov. Phys. Usp. 28, 104 (1985)

8. Z. Berezhiani, Phys. Lett. B 129, 99 (1983)

9. Z. Berezhiani, Phys. Lett. B 150, 177 (1985)

10. Z. Berezhiani, M.Y. Khlopov, Z. Phys. C 49, 73 (1991)

11. Z. Berezhiani, M.Y. Khlopov, Sov. J. Nucl. Phys. 51, 739 (1990)

12. Z. Berezhiani, M.Y. Khlopov, Sov. J. Nucl. Phys. 51, 935 (1990)

13. Z. Berezhiani, M.Y. Khlopov, Sov. J. Nucl. Phys. 52, 60 (1990)

14. Z. Berezhiani, M.Y. Khlopov, Sov. J. Nucl. Phys. 52, 344 (1990)

15. Z. Berezhiani, Phys. Lett. B 417, 287 (1998). arXiv:hep-ph/9609342

16. Z. Berezhiani, Nucl. Phys. Proc. Suppl. 52A, 153 (1997). arXiv:hep-ph/9607363

17. A. Anselm, Z. Berezhiani, Nucl. Phys. B 484, 97 (1997). arXiv:hep-ph/9605400

18. Z. Berezhiani, A. Rossi, Nucl. Phys. Proc. Suppl. 101, 410 (2001). arXiv:hep-ph/0107054

19. G. D'Ambrosio, G.F. Giudice, G. Isidori, A. Strumia, Nucl. Phys. B 645, 155 (2002). arXiv:hep-ph/0207036

20. Z. Berezhiani, A. Rossi, Nucl. Phys. B 594, 113 (2001). arXiv:hep-ph/0003084

21. Z. Berezhiani, A. Rossi, JHEP 9903, 002 (1999). arXiv:hep-ph/9811447

22. S.F. King, G.G. Ross, Phys. Lett. B 574, 239 (2003). arXiv:hep-ph/0307190

23. Z. Berezhiani, F. Nesti, JHEP 0603, 041 (2006), arXiv:hep-ph/0510011

24. L.E. Ibanez, G.G. Ross, Phys. Lett. B 332, 100 (1994). arXiv:hep-ph/9403338

25. V. Jain, R. Shrock, Phys. Lett. B 352, 83 (1995). arXiv:hep-ph/9412367

26. P. Binetruy, S. Lavignac, P. Ramond, Nucl. Phys. B 477, 353 (1996). arXiv:hep-ph/9601243

27. E. Dudas, C. Grojean, S. Pokorski, C.A. Savoy, Nucl. Phys. B 481, 85 (1996). arXiv:hep-ph/9606383

28. Z. Berezhiani, Z. Tavartkiladze, Phys. Lett. B 396, 150 (1997). arXiv:hep-ph/9611277

29. Z. Berezhiani, Z. Tavartkiladze, Phys. Lett. B 409, 220 (1997). arXiv:hep-ph/9612232

30. B. Belfatto and Z. Berezhiani, in preparation

31. Z. Berezhiani, G. Dvali, Phys. Lett. B 450, 24 (1999). arXiv:hep-ph/9811378

32. R.N. Cahn, H. Harari, Nucl. Phys. B 176, 135 (1980)

33. Z.G. Berezhiani, J.L. Chkareuli, Sov. J. Nucl. Phys. 52, 383 (1990)

34. S. Weinberg, Phys. Rev. Lett. 43, 1566 (1979)

35. D. Bourilkov, Phys. Rev. D 64, 071701 (2001). arXiv:hep-ph/0104165

36. S. Schael et al., ALEPH Collaboration, Eur. Phys. J. C 49, 411 (2007). arXiv:hep-ex/0609051

37. M. Tanabashi et al., Particle Data Group, Phys. Rev. D 98(3), 030001 (2018)

38. G. Feinberg, S. Weinberg, Phys. Rev. 123, 1439 (1961)

39. L. Willmann et al., Phys. Rev. Lett. 82, 49 (1999). arXiv:hep-ex/9807011

40. P. Foldenauer, J. Jaeckel, JHEP 1705, 010 (2017). arXiv:1612.07789 [hep-ph]

41. R.H. Parker et al., Science 360, 191 (2018)

42. T.D. Lee, C.N. Yang, Phys. Rev. 104, 254 (1956) 
43. I.Y. Kobzarev, L.B. Okun, I.Y. Pomeranchuk, Yad. Fiz. 3, 1154 (1966)

44. S.I. Blinnikov, M.Y. Khlopov, Sov. J. Nucl. Phys. 36, 472 (1982)

45. S.I. Blinnikov, M.Y. Khlopov, Yad. Fiz. 36, 809 (1982)

46. R. Foot, H. Lew, R.R. Volkas, Phys. Lett. B 272, 67 (1991)

47. L.B. Okun, Phys. Usp. 50, 380 (2007). (for a historical overview)

48. Z. Berezhiani, Int. J. Mod. Phys. A 19, 3775 (2004). arXiv:hep-ph/0312335

49. Z. Berezhiani, Eur. Phys. J. ST 163, 271 (2008)

50. E.K. Akhmedov, Z.G. Berezhiani, G. Senjanovic, Phys. Rev. Lett. 69, 3013 (1992)

51. R. Foot, H. Lew, R.R. Volkas, Mod. Phys. Lett. A 7, 2567 (1992)

52. R. Foot, R.R. Volkas, Phys. Rev. D 52, 6595 (1995). arXiv:hep-ph/9505359

53. Z. Berezhiani, R.N. Mohapatra, Phys. Rev. D 52, 6607 (1995). arXiv:hep-ph/9505385

54. L. Bento, Z. Berezhiani, Phys. Rev. Lett. 87, 231304 (2001). arXiv:hep-ph/0107281

55. L. Bento, Z. Berezhiani, Fortsch. Phys. 50, 489 (2002). arXiv:hep-ph/0111116

56. P. Achard et al., L3 Collaboration, Phys. Lett. B 517, 75 (2001). arXiv:hep-ex/0107015

57. S.N. Gninenko, N.V. Krasnikov, V.A. Matveev, Phys. Rev. D 87(1), 015016 (2013)

58. S.L. Glashow, Phys. Lett. 167B, 35 (1986)

59. S.N. Gninenko, Phys. Lett. B 326, 317 (1994)

60. C. Vigo, L. Gerchow, L. Liszkay, A. Rubbia, P. Crivelli, Phys. Rev. D 97(9), 092008 (2018). arXiv:1803.05744 [hep-ex]

61. K.S. Babu, R.N. Mohapatra, Phys. Rev. D 94(5), 054034 (2016). arXiv:1606.08374 [hep-ph]

62. A. Addazi, Z. Berezhiani, Y. Kamyshkov, Eur. Phys. J. C 77(5), 301 (2017). arXiv:1607.00348 [hep-ph]

63. Z. Berezhiani, D. Comelli, F.L. Villante, Phys. Lett. B 503, 362 (2001). arXiv:hep-ph/0008105

64. A.Y. Ignatiev, R.R. Volkas, Phys. Rev. D 68, 023518 (2003). arXiv:hep-ph/0304260

65. Z. Berezhiani, P. Ciarcelluti, D. Comelli, F.L. Villante, Int. J. Mod. Phys. D 14, 107 (2005). arXiv:astro-ph/0312605

66. Z. Berezhiani, S. Cassisi, P. Ciarcelluti, A. Pietrinferni, Astropart. Phys. 24, 495 (2006). arXiv:astro-ph/0507153
67. R. Cerulli et al., Eur. Phys. J. C 77(2), 83 (2017). arXiv: 1701.08590

68. A. Addazi et al., Eur. Phys. J. C 75(8), 400 (2015). arXiv:1507.04317 [hep-ex]

69. D. Barducci, M. Fabbrichesi, E. Gabrielli, Phys. Rev. D 98(3), 035049 (2018). arXiv:1806.05678 [hep-ph]

70. Z. Berezhiani, L. Bento, Phys. Rev. Lett. 96, 081801 (2006). arXiv:hep-ph/0507031

71. Z. Berezhiani, L. Bento, Phys. Lett. B 635, 253 (2006). arXiv:hep-ph/0602227

72. Z. Berezhiani, Eur. Phys. J. C 64, 421 (2009). arXiv:0804.2088 [hep-ph]

73. Z. Berezhiani, R. Biondi, P. Geltenbort, I.A. Krasnoshchekova, V.E. Varlamov, A.V. Vassiljev, O.M. Zherebtsov, Eur. Phys. J. C 78(9), 717 (2018). arXiv:1712.05761 [hep-ex]

74. Z. Berezhiani, F. Nesti, Eur. Phys. J. C 72, 1974 (2012). arXiv:1203.1035 [hep-ph]

75. L.J. Broussard et al., Proceedings of the APS Division of Particles and Fields (DPF 2017) : Fermilab, Batavia, Illinois, USA. arXiv: 1710.00767

76. Z. Berezhiani, M. Frost, Y. Kamyshkov, B. Rybolt, L. Varriano, Phys. Rev. D 96(3), 035039 (2017). arXiv:1703.06735 [hep-ex]

77. Z. Berezhiani, A.D. Dolgov, R.N. Mohapatra, Phys. Lett. B 375, 26 (1996). arXiv:hep-ph/9511221

78. Z. Berezhiani, L. Gianfagna, M. Giannotti, Phys. Lett. B 500, 286 (2001). arXiv:hep-ph/0009290

79. Z. Berezhiani, in From Fields to Strings: Circumnavigating Theoretical Physics, Eds. M. Shifman et al., Vol. 3, pp. 2147-2195 (2005). arXiv:hep-ph/0508233

80. A. Falkowski, S. Pokorski, M. Schmaltz, Phys. Rev. D 74, 035003 (2006). arXiv:hep-ph/0604066

81. Z. Chacko, H.S. Goh, R. Harnik, Phys. Rev. Lett. 96, 231802 (2006). arXiv:hep-ph/0506256

82. Z. Berezhiani, M. Chianese, G. Miele, S. Morisi, JHEP 1508, 083 (2015). arXiv:1505.04950 [hep-ph]

83. N. Arkani-Hamed, S. Dimopoulos, G.R. Dvali, Phys. Rev. D 59, 086004 (1999). arXiv:hep-ph/9807344

84. B. Belfatto, PoS EPS HEP2017, 660 (2017) 